\title{
Ação dos tutores e sua relação com 0 desempenho dos estudantes em curso de Licenciatura em Química sob a perspectiva da analítica da aprendizagem
}

\author{
Gláucia Mirian de Oliveira Souza Barbosa \\ João Batista Carvalho Nunes ${ }^{2}$ (D) (a) \\ João Bosco Chaves ${ }^{2}$ (1) @ \\ ${ }^{1}$ Secretaria de Educação de Maracanaú, Brasil; ${ }^{2}$ Universidade Estadual do Ceará (UECE), Brasil
}

Resumo. Este artigo responde aos seguintes objetivos: elaborar um modelo estatístico das ações dos tutores no AVA que interferem no desempenho dos estudantes do curso de Licenciatura em Química, na modalidade de educação a distância, da Universidade Aberta do Brasil / Universidade Estadual do Ceará (UAB/UECE), com fundamento na analítica da aprendizagem; e conhecer as ações que interferem no desempenho dos estudantes sob a perspectiva de tutores e estudantes, comparando o resultado com o modelo estatístico obtido na pesquisa. Para tanto, a metodologia está assentada no paradigma pragmático e na abordagem mista. Adotou o método estatístico, com uso da técnica de análise de regressão logística binária, suplementado pelo emprego de questionários com itens abertos e fechados, aplicados a tutores e estudantes. Ao final, foi obtido um modelo preditivo contendo 11 ações desenvolvidas pelos tutores, sendo sete que aumentam a probabilidade de aprovação dos estudantes e quatro que devem ser evitadas pelos tutores ou cuja frequência deve ser diminuída, pois reduzem a probabilidade de aprovação.

Palavras-chave: educação a distância; formação de professores; analítica da aprendizagem; tutoria.

Acción de los tutores y su relación con el desempeño de los estudiantes en curso de Licenciatura en Química desde la perspectiva de la analítica del aprendizaje Resumen. Este artículo responde a los siguientes objetivos: elaborar un modelo estadístico de las acciones de los tutores en el EVA que interfieren en el desempeño de los estudiantes del curso de Licenciatura en Química, en la modalidad de educación a distancia, de la Universidad Abierta de Brasil / Universidad Estatal de Ceará (UAB / UECE), con base en la analítica del aprendizaje; y conocer las acciones que interfieren en el desempeño de los estudiantes desde la perspectiva de tutores y estudiantes, comparando el resultado con el modelo estadístico obtenido en la investigación. Para ello, la metodología está asentada en el paradigma pragmático y en el enfoque mixto. Se adoptó el método estadístico, con el uso de la técnica de análisis de regresión logística binaria, suplementado por el empleo de cuestionarios con ítems abiertos y cerrados, aplicados a tutores y estudiantes. Al final, se obtuvo un modelo predictivo conteniendo 11 acciones desarrolladas por los tutores, siendo siete las que aumentan la probabilidad de aprobación de los estudiantes y cuatro las que deben ser evitadas por los tutores o cuya frecuencia debe ser disminuida, pues reducen la probabilidad de aprobación.

Palabras clave: educación a distancia; formación de profesores; analítica del aprendizaje; tutoría.

Tutors' action and their relation to the performance of undergraduate students in Chemistry from learning analytics perspective

Abstract. This article answers the following objectives: to elaborate a statistical model of tutors' actions in LMS that interfere in students' performance of the Chemistry Degree course, in distance education modality, at the Open University of Brazil / Ceará State University (UAB/UECE), based on learning analytics; and to know actions that interfere in students' performance from tutors' and students' perspective, comparing the result with statistical model obtained in the research. For this, the methodology is based on pragmatic paradigm and mixed approach. It adopted the statistical method, using binary logistic regression analysis, supplemented by the use of questionnaires with open and closed items, applied to tutors and students. At the end, a predictive model was 
obtained containing 11 actions developed by tutors, seven of which increase student approval probability and four that should be avoided by tutors or whose frequency should be decreased because they reduce approval probability.

Keywords: distance education; teacher education; learning analytics; tutoring.

\section{Introdução}

No Brasil, a educação a distância (EaD) é um tema bastante discutido nos últimos anos, sobretudo pelo aumento expressivo de seu percentual de matrícula. Os dados consolidados, em Sinopses Estatísticas e Resumos Técnicos, pelo Instituto Nacional de Estudos e Pesquisas Educacionais Anísio Teixeira (INEP) no Censo da Educação Superior ${ }^{1}$, denotam uma evolução no número de matrícula de 369.766 em 2007 para 1.756 .982 em 2017 (INEP, 2018a; INEP, 2018b), representando um aumento de 375,2\%.

0 maior atendimento encontra-se nos cursos de licenciatura ${ }^{2}$, expressando também, um crescimento significativo: 78.402 em 2005 para 745.611 em 2017 (INEP, 2016; INEP, 2018a). Isto sinaliza, entre outros fatores, a relevância da EaD na formação de professores da educação básica e a preocupação dos fomentadores de políticas públicas educacionais em priorizar esse grau acadêmico.

O Ministério da Educação (MEC) implantou o Sistema Universidade Aberta do Brasil (UAB), por meio do Decreto n ${ }^{\circ} 5.800$, de 8 de junho de 2006, no âmbito do Fórum das Estatais pela Educação, tendo como prioridade a formação de professores da educação básica. A intenção não foi criar outra instituição de ensino, mas, de acordo com os documentos oficiais, o objetivo do MEC foi articular as instituições em curso, criando possibilidades de levar o ensino superior público de qualidade aos municípios brasileiros que não possuíam cursos de formação superior ou àqueles em que os cursos ofertados não eram suficientes para atender toda a demanda.

${ }^{1}$ O Censo da Educação Superior é realizado anualmente pelo INEP, sendo considerado o retrato mais completo do Brasil sobre as instituições de ensino superior, seus cursos de graduação presencial ou a distância, cursos sequenciais, vagas oferecidas, inscrições, matrículas, "ingressantes" e concluintes e informações sobre docentes nas distintas modalidades de organização acadêmica e categoria administrativa. Os dados são coletados com base no preenchimento dos questionários, por parte das instituições e por importação de dados do Sistema e-MEC. Após preenchimento (pelas IES), conferência e validação dos dados, o Censo é finalizado, os dados são divulgados e a Sinopse Estatística é publicada. A última sinopse publicada da Educação Superior foi a do ano de 2017, atualizada em 20 de setembro de 2018.

${ }^{2}$ Os cursos de licenciatura, no Brasil, são destinados à formação de professores. 
Por meio da colaboração entre União (via universidades públicas) e entes federativos (governos estaduais e municipais), criaram-se centros de formação permanente denominados polos de apoio presenciais, em localidades consideradas estratégicas (Brasil, 2018a). De acordo com a Nota Técnica n 2/2018/CGPC/DED (Brasil, 2018b), da Coordenação de Aperfeiçoamento de Pessoal de Nível Superior (CAPES), nos 26 estados brasileiros, mais o Distrito Federal, há mais de 130 instituições públicas federais e estaduais ofertando cursos de graduação e pós-graduação na modalidade EaD em cerca de 800 polos UAB em diversos municípios.

Se há um crescimento considerável na busca pelos cursos a distância, a Academia precisa se preocupar com a qualidade desses e o consequente desempenho de seus estudantes, pois, paralelamente a esse fenômeno, é expressa a necessidade de profissionais capacitados não somente para elaborar e formatar os cursos, mas também para acompanhar e avaliá-los. Grande parte desses profissionais, contudo, tem como referência as experiências presenciais, as quais não podem ser transferidas integralmente para a modalidade a distância.

A equipe pedagógica que atua no planejamento e execução do curso em EaD é denominada de "polidocente". Esse termo foi cunhado por Oliveira, Mill, \& Ribeiro (2014, p. 67), "composto de poli, no sentido de multiplicidade, e docência, aquele que exerce a atividade de ensinar, responsável pela decisão pedagógica no ensino-aprendizagem, com o objetivo de melhorar esta última". Para os autores, a "polidocência virtual é, portanto, a docência realizada por um coletivo de trabalho na EaD, mediada pelas TDIC". Implica uma docência exercida por várias pessoas, um "coletivo de trabalhadores que, mesmo com formação e funções diversas, é responsável pelo processo de ensino-aprendizagem na EaD" (Mill, Ribeiro, \& Oliveira, p. 24). Essa equipe é composta pelos seguintes professores: o conteudista, o coordenador de disciplina, o formador (ou aplicador), o tutor (presencial e virtual); e, ainda, uma equipe multidisciplinar, com especialistas em mídia impressa, audiovisual, virtual, vídeo e webconferência, além de outros profissionais eventuais, projetista educacional e uma equipe coordenadora (Mill et al., 2014).

O profissional que atua diretamente com os cursistas é o tutor. Ele tem um papel fundamental no processo educacional desenvolvido no curso, pois se responsabiliza pelo acompanhamento e aprendizagem do estudante. A interação e a motivação fazem parte de suas atribuições, com vistas a evitar o desestímulo e a evasão. Assim, torna-se urgente e de suma importância, ante a responsabilidade desse profissional no fazer educativo da EaD, analisar as suas ações no ambiente virtual de aprendizagem (AVA), com o objetivo de propor melhorias na mediação pedagógica. É necessário identificar, para 
tanto, quais ações potencializam resultados positivos e quais minimizam a possibilidade de um desempenho satisfatório do estudante, podendo ocasionar seu desestímulo e evasão. Essas ações são disponibilizadas no AVA e podem ser quantificadas.

Nessa perspectiva, existe uma solução baseada em tecnologia (Nunes, 2015) denominada analítica da aprendizagem (learning analytics - LA), que, além de interpretar uma grande gama de dados, exprime a possibilidade de avaliar o progresso acadêmico, prever o desempenho futuro e identificar potenciais problemas (Johnson, Adams, \& Cummins, 2012, p. 22).

As pesquisas no campo da analítica da aprendizagem são recentes no Brasil, e o volume de trabalhos nessa área ainda é limitado, pois contorna um tema embrionário de investigação, sobretudo no País e na América Latina (Nunes, 2015; Santos, Cechinel, Nunes, \& Ochoa, 2017). 0 interesse crescente pela análise automática de dados educacionais, contudo, tem feito a procura pelo tema registrar ascensão. É possível confirmar essa informação com o resultado da busca realizada por Sales (2017), Gonçalves (2018) e Barbosa (2019) em suas pesquisas de doutorado.

O objetivo da analítica da aprendizagem, de acordo com Jonhson 170 et al. (2012, p. 22), é permitir que os professores e as escolas adaptem as oportunidades educacionais ao nível da necessidade e habilidade de cada aluno em tempo próximo do real, sendo possível intervir de maneira individual e eficaz nas necessidades dos estudantes.

Pesquisas confirmam a importância da utilização da LA nos processos educativos, com vistas a auxiliar na tomada de decisão de instituições, gestores, conteudistas, tutores e estudantes. Busca realizada de 2014 ao primeiro semestre de 2018 no Catálogo de Teses e Dissertações do Brasil ${ }^{3}$ e no Journal of Learning Analytics (JLA) ${ }^{4}$, contudo, indica que a maioria das pesquisas sobre o tema enfoca o desenvolvimento de modelos preditivos para reduzir índices de evasão (Cambruzi, 2014; Portal, 2016), identificar alunos em risco (Ferreira, 2016; Gray, McGuinness, Owende, \& Hofmann, 2016; Jayaprakash, Moody, Lauría, Regan, \& Baron, 2014; Sales, 2017; Waddington, Nam, Lonn, \& Teasley, 2016), ou ainda relacionar alguns fatores como tempo de permanência no ambiente (Andergassen, Mödritscher, \& Neumann, 2014), dados psicométricos (Gray et al, 2014), reações emocionais dos alunos (Pardos, Baker, San Pedro, Gowda, \& Gowda, 2014), métricas de discussões

${ }^{3}$ Disponível em https://catalogodeteses.capes.gov.br.

4 Periódico especializado em LA e com início em 2014, disponível em https:// learning-analytics.info/journals/index.php/JLA. 
estudantis (Schneider \& Pea, 2015), comportamentos online (Lowes, Lin, \& Kinghorn, 2015) e o engajamento em termos de hábitos de trabalho online (Dvorak \& Jia, 2016) com o desempenho acadêmico.

Chaves (2015), Aguiar (2016), Sales (2017) e Gonçalves (2018) realizaram suas pesquisas no mesmo contexto desta investigação (cursos da UAB/UECE). Embora sejam cursos diferentes, os dados se mostram na mesma estrutura e organização. Enquanto os autores estabeleceram relação entre os dados de interação dos estudantes e seus desempenhos, nesta pesquisa, os sujeitos são os tutores. Essa relação é comprovada, na pesquisa de Chaves (2015), por meio da técnica de correlação de postos de Spearman. Aguiar (2016) e Sales (2017) fizeram uso da regressão logística binária, enquanto Gonçalves (2018) empregou a análise de regressão logística multinomial.

Na busca de articular o tema da LA com a ação dos tutores, Oliveira (2016) e Zapparolli (2016) preocuparam-se em elaborar e utilizar ferramentas capazes de emitir relatórios mais completos da ação dos tutores e acompanhamento de seus alunos, que levem em conta suas reais necessidades e auxiliem no processo de ensino e aprendizagem. Esses resultados podem auxiliar gestores de cursos em EaD a melhorarem o tempo nas tomadas de decisões, aos tutores a alcançarem melhores resultados com seus alunos, e evitarem futuras evasões, pois as informações obtidas podem também subsidiar um acompanhamento individual e efetivo pelo tutor.

Ferramenta de avaliação das ações comportamentais desses sujeitos é apresentada por Souza (2016). 0 autor avaliou os comportamentos de tutores e turmas e identificou quais comportamentos dos tutores podem ser associados tanto de maneira positiva ou negativa com os comportamentos da turma.

Os estudos de Knight, Brozina, \& Novoselich (2016), Leeuwen (2015) e McCoy \& Shih (2016) voltaram sua atenção para os tutores como produtores de dados analíticos, e não somente consumidores. Os resultados dessas pesquisas sugerem que as ferramentas de LA podem ensejar informações agregadas a um nível administrável, possibilitando ao tutor monitorar as atividades dos estudantes e, ainda, melhorar a sua prática.

Modelo de referência para a analítica da aprendizagem é desenhado por Chatti, Dyckhoff, Schroeder, \& Thüs (2012) privilegiando quatro dimensões. Dentre estas temos o "por quê", incluindo como possíveis objetivos da LA o monitoramento, análise, predição, intervenção, tutoria/tutoria, avaliação, feedback, adaptação, personalização, recomendação e reflexão (Chatti et al., 2012, p. 9). 
Na predição, o objetivo é desenvolver, com amparo nas atividades que estão sendo realizadas pelo estudante, um modelo que preveja o seu desempenho, o qual pode ajudar na intervenção e na tomada de decisão para sugerir melhorias no processo (Chatti et al., 2012). A analítica da aprendizagem nos auxilia, deste modo, na identificação de um modelo preditivo com vistas a uma intervenção tutorial mais direcionada e efetiva.

É nesse contexto que se insere nosso objetivo geral da pesquisa: analisar as ações realizadas pelos tutores no AVA Moodle e sua relação com o desempenho dos estudantes do curso de Licenciatura em Química da Universidade Estadual do Ceará (UECE) por meio do Sistema UAB, doravante denominado UAB/UECE, tomando por base a analítica da aprendizagem. Neste artigo, centramo-nos nos seguintes objetivos específicos: elaborar um modelo estatístico das ações dos tutores no AVA que interferem no desempenho dos estudantes do curso de Licenciatura em Química da UAB/UECE, com fundamento na analítica da aprendizagem; e conhecer as ações que interferem no desempenho do estudante sob a perspectiva de tutores e estudantes, comparando o resultado com o modelo estatístico obtido na pesquisa.

Além desta introdução, o artigo traz o caminho metodológico definido para alcançar os resultados. Em seguida, encontram-se os achados da pesquisa. Ao final, são delineadas as conclusões do estudo.

\section{Caminho metodológico}

Optamos pelo modelo pragmático para fundamentar o nosso trabalho. O estudo tem um caráter prático à medida que exprime um resultado que pode influenciar diretamente na prática docente do tutor, e consequentemente, na melhoria do desempenho dos estudantes. Aproximamo-nos, ainda, desse paradigma, ao imprimir um status de integração para as abordagens quantitativas e qualitativas, aceitando qualquer possibilidade e entendendo que o casamento das abordagens só pode ser feito quando essa é a que melhor pode ajudar a responder os objetivos da pesquisa.

O pragmatismo é normalmente associado com a abordagem mista de pesquisa, pois seu foco está nas consequências da busca, na importância fundamental da questão formulada, e no uso de múltiplos métodos de coleta de dados para informar os problemas que estão sendo estudados; portanto, é pluralista e orientado para o "que funciona" e para a prática. Assim, fizemos essa opção, uma vez que nela o pesquisador coleta e analisa dados qualitativos e quantitativos em única pesquisa ou em múltiplas fases de um programa de 
estudo, e os mistura, combinando-os e priorizando um ou ambos os formatos de dados, e estruturando-os de acordo com visões de mundo filosóficas e lentes teóricas (Creswell \& Clark, 2013).

Na nossa pesquisa, os elementos quantitativos têm prioridade relativa sobre os elementos qualitativos. Com amparo nos dados de interação no AVA Moodle dos tutores do Curso de Licenciatura de Química da UAB/UECE, estabelecemos a relação com o desempenho dos estudantes. 0 resultado do primeiro momento é o modelo preditivo, obtido por meio do método estatístico, com uso da técnica de análise de regressão logística binária, contendo as ações dos tutores com coeficientes de probabilidade significativos estatisticamente para a aprovação dos estudantes.

Com esteio nesse modelo preditivo, aplicamos um questionário com tutores e estudantes do curso, por meio do aplicativo Formulários Google ${ }^{5}$. Inicialmente, procuramos captar as ações realizadas no AVA que, na visão deles, interferiam no desempenho do estudante. Em seguida, apresentamos o modelo preditivo e questionamos se eles concordavam que tais ações influenciavam na aprovação dos alunos. 0 elemento qualitativo do segundo momento tem um caráter suplementar, porquanto ajudou a compreender melhor o resultado inicial.

Elegemos o Curso de Licenciatura em Química (turma 2017.1) para realizarmos a pesquisa. Essa delimitação se deu pelo fato de esse curso ter indicado, na sua primeira turma (2009.1), baixo índice de evasão. Enquanto os cursos de Licenciatura em Física e Licenciatura em Matemática, respectivamente, alcançaram $45 \%$ e $21,8 \%$ de formandos, o curso de Licenciatura em Química chegou a 69,2\% de concludentes do total de matriculados das turmas de 2009.1 dos polos de Mauriti e Orós (Vidal, 2017).

Esta pesquisa abrangeu os três primeiros semestres do curso em cinco polos (Maracanaú, Mauriti, Beberibe, Camocim e Piquet Carneiro). 0 polo de Orós não pôde ser inserido em virtude da ausência, no momento da coleta das notas, dos diários de duas disciplinas, não sendo possível relacionar a ação dos tutores com o desempenho dos estudantes nessas disciplinas. Não incluímos o $4^{\circ}$ semestre porque a organização dos dados e da análise ocorreu enquanto esse ainda estava sendo cursado pelos estudantes.

Utilizamos um script para produzir uma planilha do banco de dados do AVA Moodle do Curso de Licenciatura em Química, em conformidade com os passos indicados em Chaves (2015). Identificamos todos os tutores.

${ }^{5}$ O Formulários Google ou Google Forms é um aplicativo web de criação e administração de questionários online. 
Separamos os tutores a distância (TD) e os presenciais (TP), pois, mesmo sendo a mediação no ambiente virtual uma responsabilidade apenas do tutor a distância, foi constatada a proeminente participação dos tutores presenciais no AVA. Todas as informações da planilha foram codificadas, garantindo-se anonimato aos sujeitos.

Em seguida, organizamos os resultados (aprovado ou não aprovado) dos alunos por polos e disciplinas. Inicialmente, foi necessário replicar cada ação do tutor para o número de alunos, por disciplina. Por exemplo, se na disciplina 1 do semestre 1 (S1_D1) havia 50 alunos matriculados, a ação do tutor (TD e/ou TP) é realizada para toda a turma, embora cada aluno tenha um desempenho diferente e, consequentemente, um resultado específico (aprovado ou não aprovado). Fizemos isso em todos os polos, semestres, disciplinas e tutores para cada aluno. A planilha final ficou com 2.625 linhas e 56 colunas.

0 próximo passo foi proceder à análise. É a segunda etapa proposta por Chatti et al. (2012) - analítica e ação - momento de explorar os dados e extrair significado com apoio neles. Reduzimos os fenômenos a termos quantitativos e manipulação estatística, permitindo comprovar relações entre si e generalizar sobre a sua natureza, ocorrência ou significado (Lakatos \& Marconi, 1991).

Valemo-nos da análise de regressão logística binária, que "tem como objetivo principal estudar a probabilidade de ocorrência de um evento definido por $Y$ que se apresenta de forma dicotômica (...), com base no comportamento de variáveis explicativas" (Fávero, 2015, p. 104). A variável dependente é o resultado do estudante (aprovado é o evento de interesse $=1$; e não aprovado é a ocorrência do não evento $=0$ ). As variáveis independentes são as ações dos tutores no ambiente (acesso, participação nos fóruns, envio de mensagens aos estudantes, feedback de atividades etc., representadas pela quantidade de vezes que esta é registrada), o sexo ( 1 = masculino e 2 = feminino) e a função ( 1 = tutor a distância e 2 = tutor presencial). Foi utilizado o software livre de análise estatística $\mathrm{R}$.

Com base no resultado do modelo preditivo, elaboramos os questionários, com questões abertas e fechadas, que foram aplicados, por meio do aplicativo Formulários Google, junto a tutores e estudantes do curso. 
A pré-testagem dos questionários foi realizada no polo de Maracanaú, com quatro tutores e cinco estudantes dos Cursos de Licenciatura em Ciências Biológicas e Física da UAB/UECE, com características próximas ao curso pesquisado. Após a aplicação do pré-teste, não se observou a necessidade de se fazer nenhum ajuste nos instrumentos.

O universo pesquisado abrangeu cinco polos (Maracanaú, Mauriti, Beberibe, Camocim e Piquet Carneiro), nove tutores (quatro a distância e cinco presencias, pois uma tutora a distância é responsável por dois polos), e 100 alunos. Responderam ao questionário sete tutores e 49 estudantes.

Ao aceitarem participar da pesquisa, tutores e estudantes assinaram um Termo de Consentimento Livre e Esclarecido (TCLE), conforme Resolução CNS no 510/2016. Utilizamos essa Resolução, entendendo, contudo, que ela ainda não satisfaz a área de Ciências Humanas, Sociais e Sociais Aplicadas (Duarte, 2015). Além disso, "pesquisa com banco de dados, cujas informações são agregadas, sem a possibilidade de identificação individual" não necessitam ser registradas e avaliadas pelo sistema CEP/CONEP (Brasil, 2016 , art. $1^{\circ}$ ).

Dentre outros pontos, oferecemos a tutores e estudantes o modelo preditivo obtido por meio da análise de regressão logística binária. Eles apontaram, tanto de modo livre, como direcionado (modelo preditivo), as ações, que, em suas perspectivas, influenciavam no desempenho do estudante.

Para chegar ao modelo preditivo final, aplicamos a análise de regressão logística nos dois modelos baseados nas ações destacadas pelos sujeitos da pesquisa. Conforme sugerido por Fávero (2015), fizemos a análise de sensibilidade, escolhendo um ponto de corte (cutoff), calculando a Eficiência Global do Modelo (EGM), a sensitividade e a especificidade. Desenhamos a curva ROC e calculamos a área sob a curva ROC, com o objetivo de perceber a eficiência do modelo para fins de previsão, de igual modo como realizamos com o modelo preditivo inicial. Aplicamos ainda o pseudo $\mathrm{R}^{2}$ de McFadden nos três modelos, com o objetivo de obter mais elementos que subsidiassem a escolha do modelo final.

Esse ajuste realizado, ou refinamento do modelo, como preferimos chamar, se insere na etapa de pós-processamento dos dados, que é responsável pela melhoria contínua no processo de analítica da aprendizagem, pois envolve a inclusão e exclusão de dados e atributos, redefinição de indicadores e identificação de novos métodos de análise (Chatti et al., 2012).

No próximo módulo deste artigo, destacaremos os resultados associados aos objetivos específicos explicitados na parte introdutória. 


\section{Resultados e discussão}

Trazemos aqui os resultados e discussões do estudo. Detemo-nos em responder aos objetivos específicos, indicando inicialmente, o modelo preditivo, resultado da análise de regressão logística binária; em seguida, as ações que interferem no desempenho do estudante sob a perspectiva dos tutores e estudantes; e, por fim, a comparação dos dois modelos baseados nas ações propostas pelos sujeitos com o modelo estatístico obtido na pesquisa.

\subsection{0 modelo preditivo}

Como explicitado no segmento imediatamente anterior, os dados do AVA Moodle foram organizados para a realização da análise de regressão logística binária. $O$ resultado apontado pelo software $\mathrm{R}$ indica os coeficientes do intercepto e de cada umas das ações consideradas estatisticamente significativas.

Para que fosse atendido um pressuposto básico da técnica de análise de regressão logística binária, que é a ausência de multicolinearidade das variáveis explicativas (Fávero, 2015), realizamos o procedimento stepwise (resultado apresentado na Tabela 1). Para definir a significância estatística de cada parâmetro (constante e coeficientes das variáveis), utilizamos a estatística z de Wald. No R, um nível de significância de 0,05 ou menor, denotando que o coeficiente da variável ou a constante é estatisticamente significante, é acompanhado por um ou mais asteriscos "*”.

Tabela 1. Resultado da regressão logística binária no $\mathrm{R}$ com $\operatorname{Pr}(>|z|)$ menor que 0,05

\begin{tabular}{cccccc}
\hline & Coefficients & & & \\
\cline { 2 - 6 } & Estimate & Std. Error & z value & $\operatorname{Pr}(>|\mathrm{z}|)$ & \\
\hline (Intercept) & 0.594321 & 0.075967 & 7.823 & $5.14 \mathrm{e}-15$ & $* * *$ \\
A06 & 0.007161 & 0.001750 & 4.092 & $4.28 \mathrm{e}-05$ & $* * *$ \\
A08 & 0.008035 & 0.001137 & 7.069 & $1.56 \mathrm{e}-12$ & $* * *$ \\
A15 & 0.114503 & 0.030949 & 3.700 & 0.000216 & $* * *$ \\
A17 & -0.007751 & 0.001872 & -4.140 & $3.47 \mathrm{e}-05$ & $* * *$ \\
A21 & -0.005325 & 0.001387 & -3.840 & 0.000123 & $* * *$ \\
A27 & 1.343030 & 0.528360 & 2.542 & 0.011026 & $*$ \\
A29 & 0.413054 & 0.144348 & 2.862 & 0.004216 & $* *$ \\
A36 & -0.065711 & 0.021479 & -3.059 & 0.002218 & $* *$ \\
A40 & 0.653014 & 0.160122 & 4.078 & $4.54 \mathrm{e}-05$ & $* * *$ \\
A50 & -0.154720 & 0.027514 & -5.623 & $1.87 \mathrm{e}-08$ & $* * *$ \\
A51 & 2.339297 & 0.716788 & 3.264 & 0.001100 & $* *$ \\
\hline S18
\end{tabular}

Signif. codes: 0 ‘***’ 0.001 ‘**’ 0.01 “*’ 0.05 ‘’ 0.1 “’ 1

(Dispersion parameter for binomial family taken to be 1 )

Null deviance: 3085.5 on 2623 degrees of freedom

Residual deviance: 2895.5 on 2612 degrees of freedom

AIC: 2919.5

Number of Fisher Scoring iterations: 4 
Dentre as 52 ações que podem ser aplicadas pelos tutores, acrescentando-se a essas as variáveis sexo e função dos tutores (a distância e presencial), totalizando 54 variáveis, apenas 11 demonstraram significância em relação ao desempenho do estudante, ou seja, ações que podem aumentar ou diminuir a probabilidade de aprovação dos alunos. São elas: A06, A08, A15, A17, A21, A27, A29, A36, A40, A50, A51. Essas variáveis representam as ações no AVA descritas na Tabela 2.

Tabela 2. Descrição das ações dos tutores significativas, conforme modelo de regressão logística

\begin{tabular}{cl}
\hline Código da ação & Descrição das ações \\
\hline A06 & Atribuir nota ao usuário \\
A08 & Visualizar curso \\
A15 & Baixar todos os arquivos \\
A17 & Conceder prorrogação para um usuário \\
A21 & Visualizar tabela de notas \\
A27 & Enviar feedback ao usuário \\
A29 & Baixar ou transferir arquivo de pasta \\
A36 & Atualizar ou modificar fórum \\
A40 & Revisar tentativa de questionário \\
A50 & Visualizar relatório de log \\
A51 & Visualizar esboço de relatório \\
\hline
\end{tabular}

Para elaborarmos a equação do modelo preditivo, utilizamos a função logística $p=1 /\left(1+e^{-2}\right)$. Assim, a equação resultante do nosso banco de dados, com as ações que devem ser consideradas pelos tutores, é a seguinte:

$$
\mathrm{p}=\frac{1}{1+e^{-\left(\alpha+\beta_{1} * A 06+\beta_{2} * A 08+\beta_{3} * A 15+\beta_{4} * A 17+\beta_{5} * A 21+\beta_{6} * A 27+\beta_{7} * A 29+\beta_{8} * A 36+\beta_{9} * A 40+\beta_{10} * A 50+\beta_{11} * A 51\right)}}
$$

Onde:

$$
\begin{aligned}
& a=+0,594321 ; \beta_{1}=+0,007161 ; \beta_{2}=+0,008035 ; \beta_{3}=+0,114503 ; \\
& \beta_{4}=-0,007751 ; \beta_{5}=-0,005325 ; \beta_{6}=+1,343030 ; \beta_{7}=+0,413054 ; \\
& \beta_{8}=-0,065711 ; \beta_{9}=+0,653014 ; \beta_{10}=-0,154720 ; \beta_{11}=+2,339297
\end{aligned}
$$

A probabilidade média estimada de um estudante ser aprovado, se o tutor realizar essas ações no ambiente, é representada pelo coeficiente da equação relacionado a cada ação. Vale destacar que o sexo e a função dos tutores não contribuíram significativamente para o modelo. Significa que a probabilidade estatística de aprovação dos estudantes independe do sexo e da função de tutoria. Na prática, a atuação do tutor a distância deveria ser 
mais significativa do que a do tutor presencial, pois a interação no ambiente virtual é incumbência desse tipo de tutoria; contudo, os dados estatísticos mostraram que o tutor presencial também tem marcante atuação no AVA.

0 modelo preditivo também nos possibilita estimar a chance de o estudante ser aprovado em uma disciplina caso o tutor realize tais ações. Comumente chance e probabilidade são utilizados como sinônimos; contudo, "seus conceitos são diferentes" (Fávero, 2015, p. 105). A chance de ocorrência de um evento, que em nosso caso é a aprovação dos estudantes, é a probabilidade de ocorrência desse evento dividida pela probabilidade da não ocorrência do mesmo evento. Assim, se a probabilidade de aprovação é de $80 \%$, por conseguinte, são as chances de aprovação de 4 para 1.

Para definirmos a chance de um aluno ser aprovado em função de uma determinada ação realizada pelo tutor, precisamos calcular o fator de chance (odds ratio). Ele nos diz como, em média, a chance de ser aprovado se modifica em função do acréscimo de uma unidade em cada variável, mantidas as demais constantes; ou seja, como as ações individualmente influenciam na chance de aprovação, se aumentam ou diminuem a chance de 0 aluno ser aprovado, mantidas as demais constantes. A Tabela 3 encerra esses percentuais para cada variável.

Tabela 3. Fator de chance (odds ratio) e porcentagem de aumento/diminuição na chance para cada variável do modelo preditivo

\begin{tabular}{cccc}
\hline Ação & Coeficiente & Fator de Chance & $\%$ \\
\hline A06 & 0,007161 & 1,007186701 & 0,7187 \\
A08 & 0,008035 & 1,008067367 & 0,8067 \\
A15 & 0,114503 & 1,121316005 & 12,1213 \\
A17 & $-0,007751$ & 0,992278962 & $-0,7721$ \\
A21 & $-0,005325$ & 0,994689153 & $-0,5311$ \\
A27 & 1,343030 & 3,830632756 & 283,06 \\
A29 & 0,413054 & 1,511426641 & 51,1427 \\
A36 & $-0,065711$ & 0,936401445 & $-6,3599$ \\
A40 & 0,653014 & 1,921322978 & 92,1322 \\
A50 & $-0,154720$ & 0,856655007 & $-14,3345$ \\
A51 & 2,339297 & 10,37394112 & 937,3941 \\
\hline
\end{tabular}


Podemos perceber que os coeficientes das ações A06, A08, A15, A27, A29, A40 e A51 agem positivamente na probabilidade de aprovação. Os percentuais expressos na Tabela 3 demonstram que, mantidas as demais condições constantes, a chance de o aluno ser aprovado aumenta, em média, quando o tutor acrescenta mais uma ação de:

- atribuir nota ao usuário (A06), 0,72\%;

- visualizar o curso (A08), 0,81\%;

- baixar todos os arquivos (A15), 12,12\%;

- envio de feedback ao usuário (A27), 283,06\%;

- baixar ou transferir arquivo de pasta (A29), 51,14\%;

- revisar tentativa de questionário (A40), 92,13\%;

- visualizar esboço de relatório (A51), 937,39\%.

É notório o fato de que as três primeiras ações têm um pequeno percentual de influência, ao passo que as quatro últimas influenciam fortemente as chances de o aluno ser aprovado. Ademais, A27 e A51, que são o envio de feedbacks aos usuários e a visualização de esboços de relatórios, mostram elevados percentuais. Significa que os tutores devem dar máxima atenção a essas ações, evidenciando e potencializando estratégias que levem os alunos a se sentirem acompanhados no decorrer do curso. É possível perceber que, mesmo com menores percentuais de fator de chance, o fato relacionado à atenção que o tutor deve dar ao aluno em seu percurso, apontando para a ideia de que o aluno, mesmo tendo autonomia para estudar nos cursos em EaD, precisa sentir-se apoiado e acompanhado para que tenha bons resultados em seu desempenho.

A Tabela 3 também evidencia que os coeficientes das ações A17, A21, A36 e A50 agem negativamente na probabilidade de aprovação. Tal significa que a chance de o aluno ser aprovado diminui, em média, quando o tutor acrescenta mais uma ação de:

- conceder prazo ao usuário (A17), 0,77\%;

- visualizar a tabela de notas (A21), 0,53\%;

- atualizar ou modificar fórum (A36), 6,36\%;

- visualizar relatório de $\log$ (A50), 14,33\%.

Embora sejam percentuais que não parecem significativos, à medida que o tutor concede prazo ao usuário e visualiza a tabela de notas, ele diminui as chances de aprovação do estudante. De igual modo, acontece quando ele atualiza ou modifica um fórum e visualiza os relatórios de log. 
Tais ações podem refletir negativamente no desempenho do estudante. Por exemplo, o fato de um tutor conceder mais prazo em uma disciplina pode levar os alunos a se acostumarem a não cumprir prazos, o que pode prejudicá-los depois em outra disciplina, perdendo uma atividade, caso não haja prorrogação; o fato de atualizar ou modificar um fórum pode ensejar insegurança nas respostas dos alunos, alterando a interpretação do que era solicitado no texto original do fórum.

É importante expressar que, embora tenhamos analisado o fator de chance para cada variável isoladamente, o resultado positivo no desempenho do estudante depende da adequação do modelo preditivo. Por isso, após estimarmos esse modelo de probabilidade de ocorrência do evento, elaboramos sua análise de sensibilidade, a qual classifica os dados/observações, com base em suas probabilidades estimadas, em eventos e não eventos, com suporte num determinado cutoff. Segundo Fávero (2015, p. 124), o cutoff,

\begin{abstract}
que nada mais é do que um ponto de corte que o pesquisador escolhe, é definido para que sejam classificadas as observações em função das suas probabilidades calculadas e, desta forma, é utilizado quando há o intuito de se elaborarem previsões de ocorrência do evento para observações não presentes na amostra com base nas probabilidades das observações presentes na amostra.
\end{abstract}

Definimos um cutoff de 0,5, que é o valor padrão geralmente utilizado em regressão logística, a exemplo de Aguiar (2016) e Sales (2017), porque estamos a estimar a probabilidade de aprovação dos estudantes do Curso de Licenciatura em Química da UAB/UECE, com amparo nas ações desenvolvidas pelos tutores. Nosso interesse, portanto, é a maximização de acertos para a classificação das observações consideradas como evento (sensitividade) e uma eficiência geral do modelo acima de 70\%.

No caso da pesquisa, das 2.624 observações, 1.968 foram classificadas corretamente, para um cutoff de 0,5, sendo que 1.864 delas eram evento (aprovação) e de fato foram classificadas como tal, e outras 84 não eram evento (não aprovação) e não foram classificadas como evento (ver Tabela 4).

Tabela 4. Classificação das observações em evento e não evento ( cutoff $=0,5$ )

\begin{tabular}{lccc}
\hline Classificação & Incidência real do evento & $\begin{array}{c}\text { Incidência real do não } \\
\text { evento }\end{array}$ & Total \\
\hline Evento & 1864 & 637 & 2501 \\
Não Evento & 39 & 84 & 123 \\
Total & 1903 & 721 & 2624 \\
\hline
\end{tabular}


A análise de sensibilidade é identificada ao se observar a eficiência global do modelo (EGM), a sensitividade e a especificidade. Para um determinado cutoff escolhido, que no nosso caso foi 0,5 , a eficiência global do modelo (EGM) diz respeito ao percentual total de acerto da classificação; a sensitividade corresponde ao percentual de acerto, considerando-se apenas as observações que de fato são evento; e a especificidade refere-se ao percentual de acerto levando-se em conta apenas as observações que não são evento.

Considerado o cutoff de 0,5 , o modelo de regressão logística binária resultante possui uma eficiência global do modelo de $74,24 \%$ (observações classificadas corretamente), a sensitividade de $97,95 \%$ (observações que foram classificadas como evento corretamente), e a especificidade de $11,65 \%$ (observações que não foram evento classificadas corretamente). Significa que mais de $70 \%$ das observações foram classificadas corretamente, e as classificações do evento, que são as aprovações, alcançaram mais que $90 \%$. De acordo com Fávero (2015, p. 155), "quanto maior o percentual de sensitividade, é bom para prever o evento".

Para analisar a qualidade do ajuste do modelo, precisamos verificar a área abaixo da curva ROC. A Figura 1 traz a curva ROC de nosso modelo. De acordo com Fávero (2015, pp. 128-129),

um determinado modelo com uma maior área abaixo da curva ROC apresenta maior eficiência global de previsão (...) quanto maior a sua convexidade em relação ao ponto $(0,1)$, maior a sua área (maior sensitividade e maior especificidade) e, consequentemente, melhor o modelo estimado para efeitos de previsão.

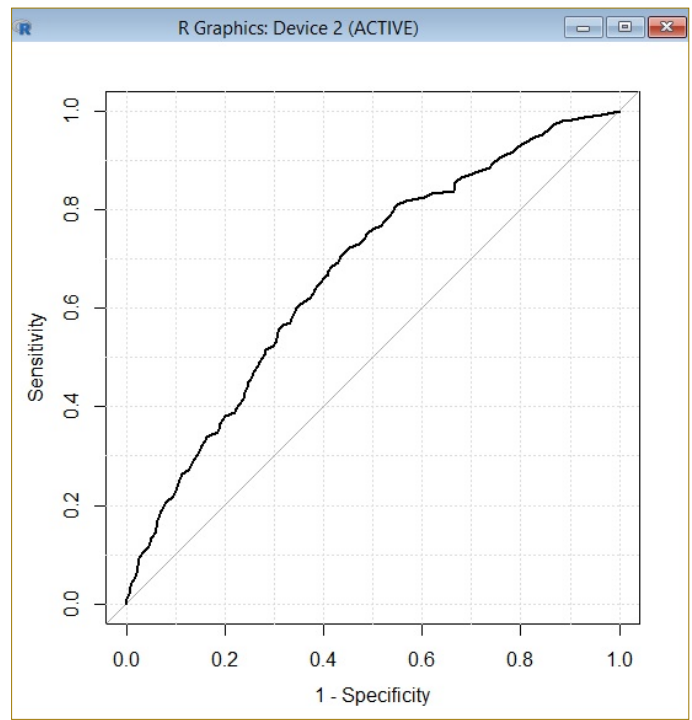

Figura 1. Curva ROC do modelo preditivo. 
Considerando-se arredondamento para quatro casas decimais, a área sob a curva ROC (Area Under an ROC Curve - AUROC) do nosso modelo foi 0,6696 . Embora possa ser classificada como "pobre" 6 para efeito de previsão, pois ficou no intervalo 60-70, ela está mais próxima do nível "justo ou moderado", cujo intervalo é 0,70-0,80, se atentarmos para o arredondamento para uma casa decimal $(0,7)$.

Estimamos o modelo preditivo e realizamos a respectiva análise de sensibilidade, mas, além do resultado estatístico, para eleger tal modelo como algo a ser replicado e validá-lo, nós o apresentamos para os sujeitos que executam as ações e para aqueles que são o objetivo delas. Questionamos se concordavam com o que havia sido encontrado. Foram aplicados, portanto, questionários com tutores e estudantes do Curso de Licenciatura em Química da UAB/UECE, dos polos pesquisados. É o que veremos no subtópico a seguir.

\subsection{A perspectiva de tutores e estudantes}

Mostramos o modelo obtido para tutores e estudantes do curso de Licenciatura de Química da UAB/UECE, tendo em vista que eles são personagens fundamentais no processo ensino-aprendizagem na educação a distância.

Perguntamos, inicialmente, a tutores e estudantes as ações que interferiam no desempenho do aluno, livremente e, em seguida, direcionada (as 52 ações possíveis de serem aplicadas pelos tutores no AVA, depois as 11 ações do modelo preditivo). As respostas dos sujeitos foram dirigidas a ações relacionadas a acompanhamento e avaliação do estudante. Ao ser expresso o modelo preditivo a tutores e estudantes, considerando as ações citadas por mais da metade dos sujeitos, os tutores referendaram apenas seis e os estudantes nove. Tutores e estudantes indicaram modelos diferentes (ver Tabelas 5 e 6).

Para fundamentar melhor o refinamento do modelo, optamos por aplicar a regressão logística binária (procedimento stepwise) para o conjunto de ações propostas pelos tutores e pelos alunos. Percebemos que o conjunto das ações com mais da metade das indicações de tutores e estudantes era mais abrangente no grupo dos estudantes, em que apenas duas ações (A29 e A51) são desconsideradas. 0 resultado da regressão logística binária para as ações destacadas pelos estudantes mostra que seus coeficientes continuaram com significância estatística, conforme se pode observar na Tabela 7.

${ }^{6}$ Para saber mais sobre essa classificação, ver: https://bit.ly/1TZDDGS. 
Tabela 5. Ações do modelo preditivo validadas pelos tutores

\begin{tabular}{lc}
\hline \multicolumn{1}{c}{ Ação } & $\%$ \\
\hline A27 - Enviar feedback ao usuário & $100,0 \%$ \\
A06 - Atribuir nota ao usuário & $85,7 \%$ \\
A17 - Conceder prorrogação para um usuário & $71,4 \%$ \\
A36 - Atualizar ou modificar fórum & $71,4 \%$ \\
A15 - Baixar todos os arquivos & $71,4 \%$ \\
A21 - Visualizar tabela de notas & $57,1 \%$ \\
\hline
\end{tabular}

Tabela 6. Ações do modelo preditivo validadas pelos estudantes

\begin{tabular}{lc}
\hline \multicolumn{1}{c}{ Ação } & $\%$ \\
\hline A27 - Enviar feedback ao usuário & $85,7 \%$ \\
A17 - Conceder prorrogação para um usuário & $85,7 \%$ \\
A06 - Atribuir nota ao usuário & $83,7 \%$ \\
A36 - Atualizar ou modificar fórum & $77,6 \%$ \\
A21 - Visualizar tabela de notas & $69,4 \%$ \\
A40 - Revisar tentativa de questionário & $65,3 \%$ \\
A08 - Visualizar curso & $57,1 \%$ \\
A15 - Baixar todos os arquivos & $53,1 \%$ \\
A50 - Visualizar relatório de log & $51,0 \%$ \\
\hline
\end{tabular}

Tabela 7. Resultado da regressão logística binária no $\mathrm{R}$ para as ações destacadas pelos estudantes

\begin{tabular}{cccccc}
\hline & Coefficients & & & \\
\cline { 2 - 6 } & Estimate & Std. Error & z value & $\operatorname{Pr}(>|\mathrm{z}|)$ & \\
\hline (Intercept) & 0.598456 & 0.075429 & 7.934 & $2.12 \mathrm{e}-15$ & $* * *$ \\
A06 & 0.006238 & 0.001726 & 3.614 & 0.000302 & $* * *$ \\
A08 & 0.007983 & 0.001148 & 6.952 & $3.60 \mathrm{e}-12$ & $* * *$ \\
A15 & 0.113640 & 0.031239 & 3.638 & 0.000275 & $* * *$ \\
A17 & -0.010259 & 0.001702 & -6.027 & $1.67 \mathrm{e}-09$ & $* * *$ \\
A21 & -0.004186 & 0.001352 & -3.096 & 0.001963 & $* *$ \\
A27 & 1.359620 & 0.526909 & 2.580 & 0.009869 & $* *$ \\
A36 & -0.078089 & 0.021350 & -3.657 & 0.000255 & $* * *$ \\
A40 & 0.419140 & 0.113596 & 3.690 & 0.000224 & $* * *$ \\
A50 & -0.100366 & 0.020319 & -4.939 & $7.83 \mathrm{e}-07$ & $* * *$ \\
\hline
\end{tabular}

Signif. codes: 0 ‘***’ 0.001 ‘** 0.01 “*’ 0.05 ‘’ 0.1 “' 1

(Dispersion parameter for binomial family taken to be 1 )

Null deviance: 3085.5 on 2623 degrees of freedom

Residual deviance: 2915.6 on 2614 degrees of freedom

AIC: 2935.6

Number of Fisher Scoring iterations: 4 
Em seguida, para cada ação, calculamos o fator de chance e fizemos a análise de sensibilidade, elegendo um cutoff de 0,5, que, em função do espaço, não serão destacados aqui. Por fim, elaboramos a curva ROC (Figura 2) e calculamos a área sob a curva ROC para verificar a qualidade do ajuste do modelo.

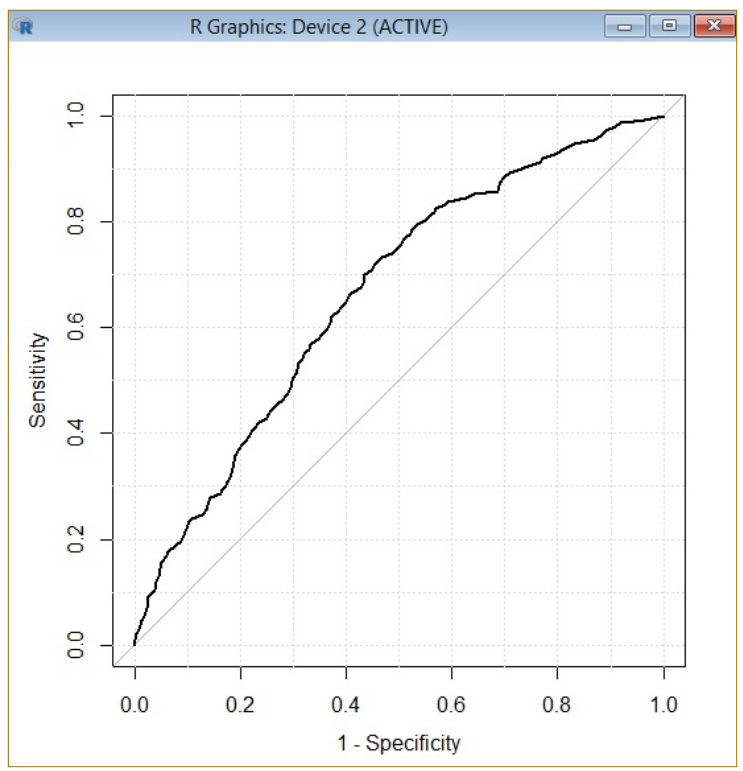

Figura 2. Curva ROC do modelo baseado nas ações destacadas pelos estudantes.

A área sob a curva ROC (AUROC) desse modelo é 0,6647. Lembramos que a área sob a curva ROC do modelo preditivo inicial foi de 0,6696 , configurando-se uma diferença muito pequena $(0,0049)$ em relação ao modelo baseado nas ações destacadas pelos estudantes.

Passando para o modelo baseado nas ações evidenciadas pelos tutores, vemos que seis são indicadas por eles. Aplicando-se a regressão logística binária, observamos que os coeficientes das ações do modelo proposto pelos tutores, exceto A06, continuaram com significância estatística, segundo se pode visualizar na Tabela 8. Por conseguinte, tal ação foi desconsiderada no modelo.

Calculamos o fator de chance para cada ação estatisticamente significante e procedemos à análise de sensibilidade, levando em conta um cutoff de 0,5. Posteriormente, elaboramos a curva ROC (Figura 3) e calculamos a área sob a curva ROC para verificar a qualidade do ajuste do modelo. 
Tabela 8. Resultado da regressão logística binária no $\mathrm{R}$ baseada nas ações destacadas pelos tutores

\begin{tabular}{|c|c|c|c|c|}
\hline & \multicolumn{4}{|l|}{ Coefficients } \\
\hline & Estimate & Std. Error & $z$ value & $\operatorname{Pr}(>|z|)$ \\
\hline (Intercept) & 0.9239951 & 0.0596302 & 15.495 & $<2 \mathrm{e}-16$ \\
\hline A15 & 0.1230624 & 0.0311903 & 3.946 & 7.96e-05 \\
\hline A17 & -0.0104108 & 0.0014882 & -6.996 & $2.64 \mathrm{e}-12$ \\
\hline A21 & 0.0017503 & 0.0004696 & 3.727 & 0.000194 \\
\hline $\mathrm{A} 27$ & 1.3439797 & 0.5242383 & 2.564 & 0.010357 \\
\hline A36 & -0.0559084 & 0.0205745 & -2.717 & 0.006580 \\
\hline
\end{tabular}

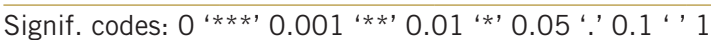

(Dispersion parameter for binomial family taken to be 1 )

Null deviance: 3085.5 on 2623 degrees of freedom

Residual deviance: 2978.1 on 2618 degrees of freedom

AIC: 2990.1

Number of Fisher Scoring iterations: 4

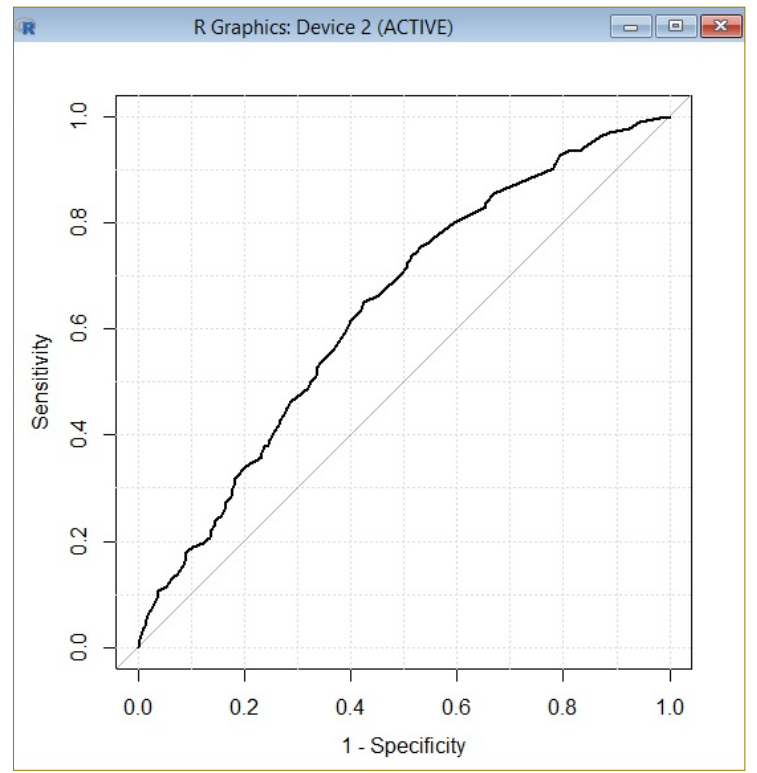

Figura 3. Curva ROC do modelo baseado nas ações destacadas pelos tutores.

A AUROC do modelo baseado nas ações destacadas pelos tutores é 0,6385 , também não se distanciando muito do modelo preditivo inicial. 0 modelo inicial, contudo, ainda expressa uma área maior do que os dois modelos baseados nas ações destacadas por tutores e estudantes.

Após obtermos tais resultados, realizamos a comparação dos três modelos para estabelecer o modelo preditivo final, conforme se pode verificar no próximo subtópico. 


\subsection{Comparação dos modelos e estabelecimento do modelo preditivo final}

Para decidir por qual modelo optar, Fávero (2015, p. 113) nos orienta no sentido de que "um maior Pseudo $\mathrm{R}^{2}$ de McFadden pode ser utilizado como critério para escolha de um modelo em detrimento de outro". Acrescenta ainda, que, contudo, "há outro critério mais adequado à escolha do melhor modelo, o qual se refere à maior área abaixo da curva ROC". Calculamos 0 Pseudo $\mathrm{R}^{2}$ de McFadden para os três modelos, a fim de melhor fundamentar nossa escolha. A síntese desses resultados encontra-se na Tabela 9.

Tabela 9. Pseudo $\mathrm{R}^{2}$ de McFadden e área sob a curva ROC dos modelos propostos

\begin{tabular}{lcc}
\hline \multicolumn{1}{c}{ Identificação do Modelo } & Pseudo R2 & AUROC \\
\hline Inicial & 0,06160539 & 0.6696 \\
Baseado nos estudantes & 0,05508018 & 0.6647 \\
Baseado nos tutores & 0,03483518 & 0.6385 \\
\hline
\end{tabular}

É perceptível o fato de que o maior Pseudo $\mathrm{R}^{2}$ de McFadden é o do modelo preditivo inicial, resultante da regressão logística binária inicial: 0,06160. Ao considerar a área sob a curva ROC do modelo preditivo inicial e dos modelos baseados nas ações destacadas pelos estudantes e pelos tutores, também verificamos que a área da predição inicial é a que denota percentual mais alto, sendo o melhor critério de escolha (Fávero, 2015).

Decidimos, portanto, que o modelo preditivo inicial permanece como o proposto para efeitos de previsão do desempenho do estudante do curso de Licenciatura em Química da UAB/UECE, constituído de 11 ações. Lembramos que existem ações que podem aumentar e outras que podem diminuir a probabilidade e chance de aprovação do estudante. No próximo segmento, são tecidas as conclusões acerca desta pesquisa.

\section{Conclusões}

Após analisarmos os três modelos obtidos na pesquisa, pudemos perceber que o modelo estatístico inicial, resultado da análise de regressão logística binária, é o mais apropriado para efeito de previsão. A análise dos resultados nos fez concluir, por conseguinte, que as ações a serem priorizadas pelos tutores do Curso de Licenciatura em Química da UAB/UECE, com o objetivo de aumentar a probabilidade de aprovação dos estudantes, são: Atribuir nota ao usuário - A06; Visualizar curso - A08; Baixar todos os arquivos - A15; Enviar feedback ao usuário - A27; Baixar ou transferir arquivo de pasta - A29; Revisar tentativa de questionário - A40; Visualizar esboço 
de relatório - A51. Essas ações possuem coeficientes positivos e fatores de chances que, mantidas as demais condições constantes, aumentam a probabilidade de aprovação do estudante.

Por outro lado, as ações que devem ser evitadas pelos tutores ou cuja frequência há que ser diminuída, pois reduzem a probabilidade de aprovação dos estudantes são: Conceder prorrogação para um usuário - A17; Visualizar tabela de notas - A21; Atualiziar ou modificar fórum - A36; e, Visualizar relatório de $\log$ - A50. Essas ações evidenciam coeficientes negativos e fatores de chance que, mantidas as demais condições constantes, diminuem a probabilidade de aprovação.

Verificamos que devem ser respeitados os prazos de retorno aos questionamentos dos estudantes e às suas atividades, pois o feedbacké muito importante para que estes permaneçam ativos no curso. Os feedbacks devem indicar os erros e acertos dos alunos, possibilitando-Ihes identificar em que aspectos precisam melhorar e os que devem continuar sendo reforçados.

Quando os tutores atribuem notas aos estudantes, estes podem ficar mais atentos e menos negligentes em relação ao ensino aprendizagem e com o curso como um todo. A probabilidade de aprovação aumenta de acordo com o fator de chance.

Ao se mostrarem vigilantes com a avaliação dos estudantes, atribuindo as notas, e revisando as tentativas de questionário, os estudantes podem perceber que os tutores estão atentos a sua trajetória no curso. Como consequência, podem ficar mais propensos a atenderem as expectativas lançadas pelo curso, a disciplina e o tutor.

Os relatórios são utilizados, especialmente, para dar ciência ao coordenador do curso sobre a situação dos seus cursistas. Esses registros, contudo, possibilitam ao tutor uma visão geral do estado da sua turma e, se for necessário, de alunos, individualmente. Portanto, a visualização do esboço de relatório deve ser valorizada e mais bem utilizada pelos tutores. Os coeficientes, percentuais de significância e fator de chance do esboço de relatório comprovam tal asserção.

O fato de conceder prorrogação de prazo para o envio das atividades pelos alunos diminui suas chances de aprovação. Assim, deve ser menos aplicada pelos tutores. Embora os estudantes acreditem ser positiva para o seu desempenho, essa ação pode levar os alunos a se acostumarem a não cumprir prazos, o que pode prejudicá-los depois em outra disciplina, perdendo uma atividade, caso não haja prorrogação. 
A visualização da tabela de notas, de acordo com o modelo, diminui de maneira muito pequena a probabilidade de aprovação dos estudantes. Mesmo assim, é estranho pensar que a ação de visualizar a tabela de notas por parte de um tutor possa diminuir a probabilidade de um aluno ser aprovado. Pode-se supor (algo que precisa ser investigado por outras pesquisas) que essa ação, no entanto, realizada sem uma reflexão efetiva sobre como contribuir para aqueles que estão com baixo desempenho, pode favorecer junto ao tutor a cristalização de perfis de alunos que tenderão a ser bem avaliados e aqueles que continuarão a não receber essa atenção.

O fórum é uma das atividades mais utilizadas nos cursos em EaD; contudo, ao criar um fórum, o tutor precisa estar atento para que este não precise de atualizações, pois, à medida que o tutor faz uma modificação, ele diminui as chances de aprovação dos alunos. 0 fato de atualizar ou modificar um fórum pode ensejar insegurança nas respostas dos alunos, alterando a interpretação do que era solicitado no texto original do fórum.

A visualização do relatório de $\log$, segundo o modelo, contribui com a diminuição da probabilidade de aprovação dos estudantes. Nesse caso, pode-se pensar na hipótese (algo que precisa ser investigado por outras pesquisas) de que se o tutor usa seu tempo para visualizar o relatório de log, mas não efetiva estratégias para ajudar os alunos com baixas interações no AVA, essa ação apenas diminuirá o tempo que ele poderia dedicar a ações reconhecidas como de influxo positivo no desempenho discente (por exemplo, fornecer feedback aos alunos).

Os resultados obtidos evidenciam o potencial da analítica da aprendizagem para contribuir no desvelamento de fatores que tendem a influenciar a aprendizagem e o consequente desempenho de estudantes, possibilitando a constituição de estratégias que favoreçam a melhoria dos indicadores de sucesso acadêmico.

\section{Referências bibliográficas}

Aguiar, A. N. (2016). Evasão no curso de Licenciatura em Matemática a distância da UECE sob a perspectiva da analítica da aprendizagem (Dissertação de Mestrado, Universidade Estadual do Ceará, Fortaleza, Brasil). Disponível em https://bit.ly/2LthgyO

Andergassen, M., Mödritscher, F., \& Neumann, G. (2014). Practice and repetition during exam preparation in blended learning courses: Correlations with learning results. Journal of Learning Analytics, 1(1), 48-74. https://dx.doi.org/10.18608/jla.2014.11.4 
Barbosa, G. M. O. S. (2019). Ação dos tutores e sua relação com o desempenho dos estudantes em curso de licenciatura da UAB/UECE sob a perspectiva da analítica da aprendizagem (Tese de Doutorado não publicada). Universidade Estadual do Ceará, Fortaleza, Brasil.

Brasil. Ministério da Saúde. Conselho Nacional de Saúde. (2016). Resolução nº 510, de 7 de abril de 2016. Disponível em https://bit.ly/2EOqAEo

Brasil. Ministério da Educação. Coordenação de Aperfeiçoamento de Pessoal de Nível Superior. (2018a, Março 27). Oqueéa UAB?[página web]. Disponível em https://bit.ly/2Y53B2i

Brasil. Ministério da Educação. Coordenação de Aperfeiçoamento de Pessoal de Nível Superior. (2018b). Nota Técnica $n^{\circ}$ 2/2018/CGPC/DED

Cambruzzi, W. L. (2014). GVWISE: uma aplicação de learning analytics para a redução da evasão na educação a distância (Dissertação de Mestrado, Universidade do Vale do Rio dos Sinos, São Leopoldo, Brasil). Disponível em https://bit.ly/2V5PPuf

Chatti, M. A., Dyckhoff, A. L., Schroeder, U., \& Thüs, H. (2012). A reference model for learning analytics. International Journal of Technology Enhanced Learning, 4(5-6), 318-331. https://dx.doi.org/10.1504/IJTEL.2012.051815

Chaves, J. B. (2015). Formação a distância de professores em Matemática pela UAB/UECE: Relação entre interação e desempenho à luz da analítica da aprendizagem (Dissertação de Mestrado, Universidade Estadual do Ceará, Fortaleza, Brasil). Disponível em https://bit.ly/2LBKonE

Creswell, J. W., \& Clark, V. L. P. (2013). Pesquisa de métodos mistos (2 ed.). Porto Alegre: Penso.

Duarte, L. F. D. (2017). Cronologia da luta pela regulação específica para as Ciências Humanas e Sociais da avaliação da ética em pesquisa no Brasil. Práxis Educativa, 12(1), 267-286. https://dx.doi.org/10.5212/PraxEduc.v.12i1.0015

Dvorak, T., \& Jia, M. (2016). Online work habits and academic performance. Journal of Learning Analytics, 3(3), 318-330. https://dx.doi.org/10.18608/jla.2016.33.15

Fávero, L. P. (2015). Análise de dados: Modelos de regressão com Excel, Stata e SPSS. Rio de Janeiro: Elsevier.

Ferreira, J. L. C. (2016). MD-PREAD: Um modelo para predição de reprovação de aprendizes na educação a distância usando árvore de decisão (Dissertação de Mestrado, Universidade do Vale do Rio dos Sinos, São Leopoldo, Brasil). Disponível em https://bit.ly/2JIF19i

Gonçalves, M. T. L. (2018). Formação do pedagogo para a gestão escolar na UAB/UECE: $A$ analítica da aprendizagem na educação a distância(Tese de Doutorado, Universidade Estadual do Ceará, Fortaleza, Brasil). Disponível em https://bit.ly/2JnE3co

Gray, G., McGuinness, C., Owende, P., \& Hofmann, M. (2016). Learning factor models of students at risk of failing in the early stage of tertiary education. Journal of Learning Analytics, 3(2), 330-372. https://dx.doi.org/10.18608/jla.2016.32.20

Instituto Nacional de Estudos e Pesquisas Educacionais Anísio Teixeira. (2016). Sinopse estatística de educação superior 2015. Brasília: Autor. Disponível em https://bit.ly/2WpnG2Y

Instituto Nacional de Estudos e Pesquisas Educacionais Anísio Teixeira. (2018a). Sinopse estatística de educação superior 2017. Brasília: Autor. Disponível em https://bit. Iy/2CAsUQN 
Instituto Nacional de Estudos e Pesquisas Educacionais Anísio Teixeira. (2018b). Censo da educação superior. Notas estatísticas 2017. Brasília: Autor. Disponível em https:// bit.ly/2Ri1fK5

Jayaprakash, S. M., Moody, E. W., Lauría, E. J. M., Regan, J. R., \& Baron, J. D. (2014). Early alert of academically at risk students: an open source analytics initiative. Journal of Learning Analytics, 1(1), 6-47. https://dx.doi.org/10.18608/jla.2014.11.3

Johnson, L., Adams, S., \& Cummins, M. (2012). The NMC horizon report: 2012 Higher education edition. Austin, Texas: The New Media Consortium. Disponível em https://bit.Iy/2VTklfg

Knight, D. B., Brozina, C., \& Novoselich, B. (2016). An investigation of first-year engineering student and instructor perspectives of learning analytics approaches. Journal of Learning Analytics, 3(3), 215-238. https://dx.doi.org/10.18608/jla.2016.33.11

Lakatos, E. M., \& Marconi, M. A. (1991). Metodologia científica (2. ed.). São Paulo: Atlas.

Leeuwen, A. (2015). Learning analytics to support teachers during synchronous CSCL: Balancing between overview and overload. Journal of Learning Analytics, 2(2), 138-162. https:// dx.doi.org/10.18608/jla.2015.22.11

Lowes, S., Lin, P., \& Kinghorn, B. (2015). Exploring the link between online behaviours and course performance in asynchronous online high school courses. Journal of Learning Analytics, 2(2), 169-194. https://dx.doi.org/10.18608/jla.2015.22.13

McCoy, C., \& Shih, P. (2016). Teachers as producers of data analytics: A case study of a teacherfocused educational data science program. Journal of Learning Analytics, 3(3), 193-214. https://dx.doi.org/10.18608/jla.2016.33.10

Mill, D. R. S., Ribeiro, L. R. C., \& Oliveira, M. R. G. (2014). Múltiplos enfoques sobre a polidocência na educação a distância virtual. In D. R. S. Mill, L. R. C. Ribeiro \& M. R. G. Oliveira (Org.), Polidocência na educação a distância: Múltiplos enfoques (pp. 13-24). São Carlos: Ed. UFSCar.

Nunes, J. B. C. (2015). Estado da arte sobre analítica da aprendizagem na América Latina. In J. B. Castro \& T. E. V. Silva (Coords.), Anais dos workshops do Congresso Brasileiro de Informática na Educação (pp. 1024-1033). https://dx.doi.org/10.5753/cbie. wcbie.2015.1024

Oliveira, E. D. S. (2016). Modelo de diagnóstico de dificuldades de aprendizagem orientado a conceitos (Dissertação de Mestrado, Universidade Federal da Paraíba, João Pessoa, Brasil). Disponível em https://bit.ly/2VnUOvl

Oliveira, M. R. G., Mill, D. R. S., \& Ribeiro, L. R. C. (2014). A gestão da sala de aula virtual e os novos saberes para a docência na modalidade de Educação a Distância. In D. R. S. Mill, L. R. C. Ribeiro \& M. R. G. Oliveira (Org.), Polidocência na educação a distância: Múltiplos enfoques (pp. 61-76). São Carlos: Ed. UFSCar.

Pardos, Z. A., Baker, R. S. J. D., San Pedro, M. O. C. Z., Gowda, S. M., \& Gowda, S. M. (2014). Affective states and state tests: Investigating how affect and engagement during the school year predict end-of-year learning outcomes. Journal of Learning Analytics, 1 (1), 107-128. https://dx.doi.org/10.18608/jla.2014.11.6

Portal, C. (2016). Estratégias para minimizar a evasão e potencializar a permanência em EaD a partir de sistema que utiliza mineração de dados educacionais e learning analytics. (Dissertação de Mestrado, Universidade do Vale do Rio dos Sinos, São Leopoldo, Brasil). Disponível em https://bit.ly/2H7helA 
Sales, V. M. B. (2017). Analítica da aprendizagem como estratégia de previsão de desempenho de estudantes de curso de Licenciatura em Pedagogia a distância(Tese de Doutorado, Universidade Estadual do Ceará, Fortaleza, Brasil). Disponível em https://bit. Iy/2H7eSJP

Santos, H. L., Cechinel, C., Nunes, J. B. C., \& Ochoa, X. (2017). An initial review of learning analytics in Latin America. In: A. Díaz, A. Casali, M. C. Rivas, \& A. S. Sprock (Eds.), 2017 Twelfth Latin American Conference on Learning Technologies (LACLO), (pp. 1-9). https://dx.doi. org/10.1109/LACLO.2017.8120913

Schneider, B., \& Pea, R. (2015). Does seeing one another's gaze affect group dialogue? A computational approach. Journal of Learning Analytics, 2(2), 107-133. https:// dx.doi.org/10.18608/jla.2015.22.9

Souza, R. C. (2016). Aplicação de learning analytics para avaliação do desempenho de tutores a distância (Dissertação de Mestrado, Universidade Federal Rural do Semi-Árido, Mossoró, Brasil). Disponível em https://bit.ly/2Ycz3f6

Vidal, E. M. (2017). Universidade Aberta do Brasil na Universidade Estadual do Ceará: Acesso, permanência e sucesso. Estudo de dados a partir do SisUab e SisRel. Fortaleza: UECE.

Waddington, R. J., Nam, S., Lonn, S., \& Teasley, S. D. (2016). Improving early warning systems with categorized course resource usage. Journal of Learning Analytics, 3(3), 263290. https://dx.doi.org/10.18608/jla.2016.33.13

Zapparolli, L. S. (2016). FAG: Ferramenta de apoio à gestão no ambiente virtual de aprendizagem Moodle utilizando técnicas de Business Intelligence (Dissertação de Mestrado, Universidade Federal do ABC, Santo André, Brasil). Disponível em https://bit. Iy/2VITnOK 